\title{
Sous-traitance et qualité dans une centrale nucléaire
}

Entretien avec Gilles Reynaud, président et de l'association « Ma zone contrôlée »

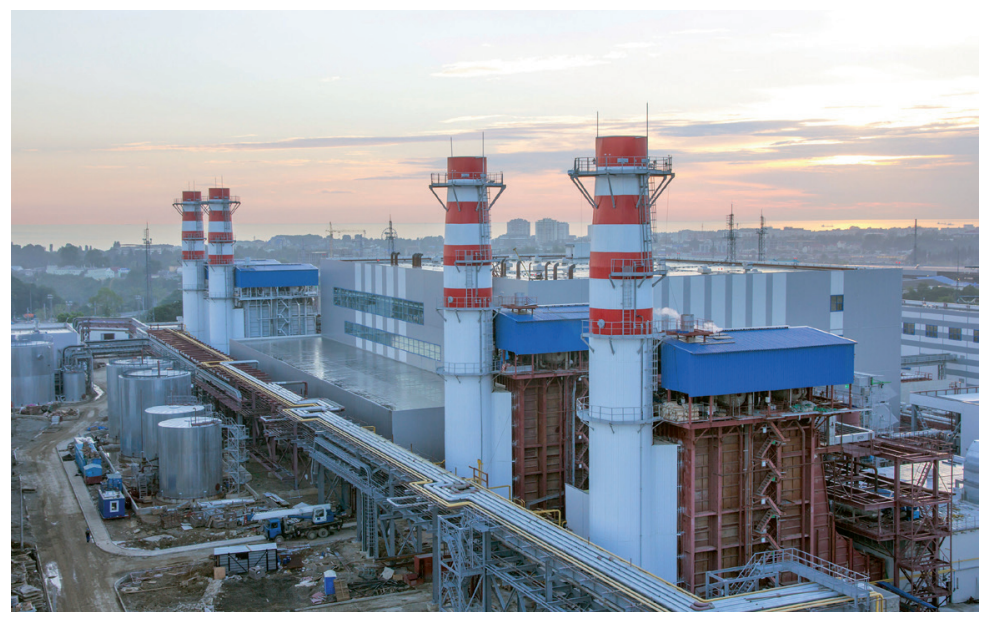

Comme dans la plupart des activités industrielles, I'industrie nucléaire a recours à la sous-traitance pour assurer I'exploitation de sa filière. Cependant, le rôle des salariés non statutaires a beaucoup évolué et comporte des spécificités quil peuvent avoir un impact sur la sureté d'une centrale. Entretien avec un sous-traitant.

\section{Quelle est l'importance de la sous-traitance dans les centrales nucléaires?}

Les grandes entreprises du secteur nucléaire (les " donneurs d'ordre ") font réaliser des tâches similaires soit par leurs propres salariés statutaires, soit par des salariés d'autres entreprises (les «sous-traitants"). À leur tour, celles-ci peuvent sous-traiter à des entreprises encore plus petites (sous-traitance « en cascade »). Les 160000 salariés des entreprises sous-traitantes jouent ainsi un rôle invisible mais capital dans la production d'électricité, exécutant 80\% des activités dans différents domaines : assainissement, maintenance, logistique, radioprotection, traitement de déchets, démantèlement.

\section{Comment évolue le domaine?}

Le vieillissement des installations nous préoccupe ; la situation économique des grands donneurs d'ordres aussi, car cela conditionne directement la durée d'attribution des marchés aux entreprises extérieures. Cette durée se situe actuellement entre 1 et 6 ans, ce qui selon nous est trop court pour des recrutements stables : cela encourage un recours massif et savamment organisé à la sous-traitance.

Notre travail réel est proche du secteur de la métallurgie ou du bâtiment-travaux publics ; or on nous applique de plus en plus la convention collective dite "Syntec » du secteur bureau d'étude-ingénierie ; elle est inappropriée, mais 30\% moins onéreuse pour l'exploitant ${ }^{(\mathrm{a})}$. De même, les salariés réalisant des opérations d'assainissement se voient appliquer la convention collective du nettoyage, moins couteuse. Ainsi, le recours aux entreprises socialement moins-disantes vise souvent à contourner les avantages sociaux des salariés statutaires des grands donneurs d'ordre.

Non seulement c'est illicite (cela s'appelle du «prêt de main d'œuvre »), mais en outre nous percevons le contrecoup direct sur le terrain de ce choix purement économique. Les salariés, renouvelés rapidement, sont démotivés et la qualité finale du travail réalisé nous semble baisser. 
$>>>$

\section{Comment la sous-traitance et la qualité sont-elles liées?}

Tout d'abord, nous mettons en avant le fait que, depuis plusieurs années, les donneurs d'ordre ne surveillent pas assez l'organisation de leurs sous-traitants. Ensuite, à force de sous-traiter et de ne pas avoir suffisamment anticipé les départs à la retraite de plusieurs cœurs de métiers clés, l'exploitant perd peu à peu, mais irrémédiablement, la maitrise de l'outil de production. Enfin, en fonction des sites où nous intervenons, des formations ou recyclages spécifiques sont dispensés aux futurs intervenants ; ainsi, auparavant, des questions essentielles portant sur la radioprotection, la sureté, la sécurité, la qualité, étaient éliminatoires : pourquoi sont-elles désormais supprimées?

Un comité a fait un très gros travail ${ }^{(b)}$ pour recenser la grande variété d'incidents, les classer, analyser leurs causes (liées à l'organisation, à la technique ou à l'humain), et proposer des améliorations. Sur le plan positif, selon l'ASN, il n'y a quasiment pas de travailleurs détachés en zone contrôlée, ni de dépassement de dose. En revanche, faute d'une bonne préparation des interventions, et suite au manque de moyens humains, les règles élémentaires sont parfois bafouées. Il y a une augmentation des incidents lors d'opérations de maintenance, des contaminations internes, des sous-traitants qui atteignent la dose limite et sont ensuite envoyés dans d'autres secteurs.

\section{Quelles leçons ont été tirées de Fukushima?}

Le retour d'expérience de l'accident a prouvé l'importance des connaissances des salariés sous-traitants d'un autre site, Fukushima daini, à 40 km de Fukushima daiichi. Ce site, également en bord de mer, avait lui aussi perdu la source d'alimentation électrique des circuits de sauvegarde. Les équipes d'intervention d'urgence de l'entreprise TEPCO étaient dans l'incapacité de réalimenter en électricité le site. Les sous-traitants, qui avaient la connaissance des installations, ont su connecter les câbles correctement.

Suite à l'accident de Fukushima, des évaluations de sureté ont été menées sur l'ensemble du parc français. Elles ont intégré le facteur humain et la sous-traitance. Elles ont abouti en 2012 à une nouveauté, le Cahier des Charges Social qui oblige les entreprises reprenant un marché à en reprendre aussi les salariés. Malgré cette protection, l'entreprise repreneuse n'est pas obligée de garder les acquis des salariés (ancienneté, qualification, convention collective). En pratique, les salariés se voient moins bien payés, moins couverts, moins reconnus dans leurs compétences et leurs expositions professionnelles, ce qui induit leur démotivation.

\section{Que proposez-vous ?}

Le lien étroit qui doit exister entre exploitant et sous-traitants, détérioré par les facteurs économiques, devrait être restauré. Par exemple, nous estimons nécessaire que les salariés sédentaires des entreprises sous-traitantes intègrent, sur la base du volontariat, les équipes des Plans d'Urgence Interne des divers exploitants. Ils sauront faire preuve de responsabilité professionnelle et d'engagement citoyen, y compris en faisant remonter à temps les alertes, et en se mobilisant lors d'un éventuel accident.

Nous demandons l'instauration, au niveau national, d'un statut spécifique et protecteur pour tous les salariés qui font le même travail. La prolongation éventuelle de durée de vie des centrales, les futurs démantèlements, dans cette filière en pleine réorganisation, ne pourront se faire qu'avec ces salariés enfin reconnus socialement.

a. [NdE] Dans une note publiée le 31 juillet 2018, EDF indique (p. 12) que selon ses calculs, le changement de convention collective représente une baisse de $12 \%$ pour les salaires les plus élevés, voire une hausse de 1,2\% pour les salaires les plus bas. Dans la même note (p. 4, p. 5 et p. 14), EDF indique que le nombre de niveaux de sous-traitance est limité à 3, et que les "Évènements significatifs sureté " sont en baisse (600 par an dont 50 imputables à des sous-traitants). Source : www.edf.fr/sites/default/files/contrib/groupe-edf/ producteur-industriel/hydraulique/Notes\%20d\%27info/note_info_pompili.pdf

b. La sous-traitance en situation de fonctionnement normal : organisation et conditions d'intervention, Comité sur les facteurs sociaux, organisationnels et humains (COFSOH), janvier 2017.
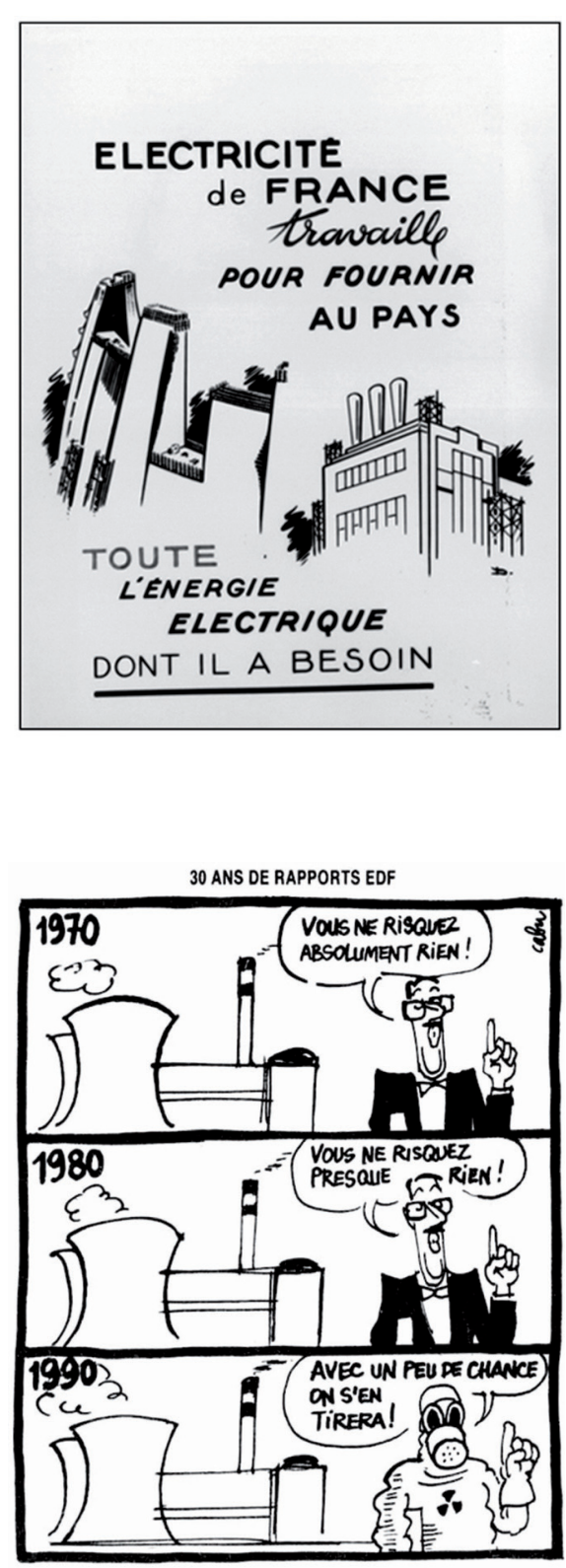

En savoir plus

- C. Dubout, Je suis décontamineur dans le nucléaire, Ed. Paulo Ramand (2010).

- G. Reynaud, dans Nucléaire et territoire, livre blanc de l'ANCCLI, janvier 2017, p. 26.

- Voir aussi le site de l'association, www.ma-zone-controlee.com Son objet : informer afin de favoriser l'échange entre les salariés, statutaires ou majoritairement sous-traitants, des industries à risques (nucléaire, chimique, pétrochimique) pour améliorer la sécurité des interventions et la sûreté des installations, pour le respect des générations futures et celui de l'environnement. 